\title{
Récits de la mise en oeuvre de modèles de réponse à l'intervention inspirée d'un modèle d'accompagnement systémique du développement pédagogique et organisationnel
}

\section{Marie-Hélène Guay et Brigitte Gagnon}

\section{Volume 7, mai 2020}

L’approche de la réponse à l'intervention et la prévention des difficultés d'apprentissage à l'école

URI : https://id.erudit.org/iderudit/1070387ar

DOI : https://doi.org/10.7202/1070387ar

\section{Aller au sommaire du numéro}

\section{Éditeur(s)}

Université Laurentienne

\section{ISSN}

1920-6275 (imprimé)

1929-8544 (numérique)

Découvrir la revue

\section{Citer cet article}

Guay, M.-H. \& Gagnon, B. (2020). Récits de la mise en oeuvre de modèles de réponse à l'intervention inspirée d'un modèle d'accompagnement systémique du développement pédagogique et organisationnel. Enfance en difficulté, 7, 153-173. https://doi.org/10.7202/1070387ar

\section{Résumé de l'article}

Au cours des dernières années, deux commissions scolaires de la Montérégie, au Québec, ont amorcé la mise en oeuvre d'un modèle de réponse à

l'intervention (RàI) pour l'apprentissage du langage oral et écrit, au préscolaire et au premier cycle du primaire, en s'inspirant d'un modèle

d'accompagnement systémique du développement pédagogique et organisationnel (Guay et Gagnon, 2020a). Dans cet article, nous présentons les récits de ces deux expériences de mises en oeuvre en détaillant des outils et des dispositifs élaborés pour les appuyer. Nous faisons également état de bons coups et d'obstacles rencontrés. Ces récits visent à guider les équipes de leaders chargées de soutenir le déploiement d'un modèle de réponse à l'intervention dans une organisation scolaire. 


\section{Récits de la mise en œuvre de modèles de réponse à l'intervention inspirée d'un modèle d'accompagnement systémique du développement pédagogique et organisationnel}

\section{Marie-Hélène Guay ${ }^{1}$ et Brigitte Gagnon ${ }^{2}$}

${ }^{1}$ Université de Sherbrooke et ${ }^{2}$ Commission scolaire des Hautes-Rivières

La mise en œuvre d'un modèle de la réponse à l'intervention (RàI) nécessite de nombreux ajustements au sein d'une organisation scolaire. Pour ce faire, les équipes-écoles, les directions d'établissements, les professionnels, les directions des services et les directions générales qui la soutiennent gagnent à se doter d'un modèle d'accompagnement conscient pour agir, éduquer et chercher des solutions. Dans cette perspective, nous avons développé un modèle d'accompagnement systémique du développement pédagogique et organisationnel (Guay et Gagnon, 2020a).

Ce modèle d'accompagnement a été développé en contexte de recherche-action (Guay, Prudhomme et Dolbec, 2016). Comme c'est souvent le cas dans la diffusion des résultats d'une recherche-action, nous utilisons ici le récit pour contextualiser et expliciter des savoirs professionnels issus d'une telle recherche (Herr et Anderson, 2015; McNiff, 2016).

L'accompagnement systémique du développement pédagogique et organisationnel est défini comme un savoir-être et un savoir-faire d'une équipe de leaders assurant la mise en cohérence progressive des 
composantes et des relations des situations pédagogiques constitutives de leur organisation scolaire. Une telle équipe est constituée d'enseignants, d'orthopédagogues, de directions d'établissements, de professionnels, de directions de services ou de membres de la direction générale qui ont développé et assument leur pouvoir d'influence. Pour accompagner le développement pédagogique et organisationnel, elle gagne à mettre en œuvre certaines actions fondamentales, dont :

1) se donner et rendre explicite une vision systémique des situations pédagogiques qu'elle accompagne;

2) conscientiser et rendre explicites ses présupposés;

3) initier, avec des volontaires, un projet-pilote lié à la vision organisationnelle et contribuant à l'enrichir;

4) soutenir le transfert de connaissances et d'outils validés issus de recherches, dont ceux générés au sein de l'organisation;

5) soutenir et encourager la collaboration pour agir et apprendre;

6) établir une communication qui soutient la compréhension réciproque et la construction d'un sens commun;

7) agir de façon authentique; et

8) être elle-même accompagnée.

Inspirées de ce modèle, des équipes de leaders de deux commissions scolaires de la Montérégie, au Québec, soutiennent la mise en œuvre de modèles RàI pour l'apprentissage du langage oral et écrit au préscolaire et au premier cycle du primaire. Le récit de tels déploiements est relaté ici et suivi d'un compte rendu de certains bons coups effectués et de certaines difficultés rencontrées, à ce jour, par ces équipes de leaders dont nous faisons ou avons fait partie.

\section{Récit de la mise en œuvre de modèles RàI à la Commission scolaire des Trois-Lacs}

La Commission scolaire des Trois-Lacs, en Montérégie, dessert plus de 16000 élèves au sein de 25 établissements au primaire, 3 établissements au secondaire et un multicentre pour la formation générale aux adultes et la formation professionnelle où œuvrent plus de 2000 employés. En 2010 , le taux de sorties sans diplôme ni qualification au secondaire et en formation générale aux jeunes était d'environ $85 \%$. L'équipe des services éducatifs, en collaboration avec la direction générale, les syndicats et les directions d'établissements, a alors entrepris plusieurs projets collaboratifs, dont l'élaboration d'une politique de formation continue 
des enseignants (CSTL, 2005), d'un référentiel des compétences des conseillers pédagogiques et d'une typologie des types d'activités de formation continue (Guay, Francoeur et Ménard, 2016). Cette dernière favorise la communication entre les équipes-écoles et les services éducatifs pour l'élaboration du plan de formation continue annuel dans chaque école (Guay, Ste-Marie, McSween, Bédard et Lecompte, 2015). La direction générale a créé un poste de coordonnatrice au développement et à la planification stratégique. Son mandat était d'accompagner les directions d'établissements et les directions de services volontaires dans le pilotage de leurs projets éducatifs dans la coordination de leurs plans de service avec le plan stratégique de la commission scolaire. Cette cadre-conseil devait aussi soutenir le transfert des connaissances issues de la recherche (CTREQ et RENARD, 2015; Guay, Francoeur et Daoust, 2014). En partenariat avec les écoles et les services, la direction générale et le comité de veille du plan stratégique ont également créé et diffusé une liste des facteurs explicatifs de la réussite dans une organisation scolaire (Guay, Smith, Ménard et Dewany, 2015). Or, l'analyse collaborative de la situation actuelle sous-jacente à l'actualisation du plan stratégique 2009-2014 (CSTL, 2009) révèle que l'organisation peine à soutenir le développement et la réussite des élèves à risque et en difficulté.

En 2011-2012, la directrice des services complémentaires et de l'adaptation scolaire coordonne la révision de la politique d'adaptation scolaire inspirée des recherches sur les modèles RàI (CSTL-SCAS, 2013). On y voit apparaître des leviers essentiels à la réussite de tous les élèves (incluant ceux à risque et en difficulté), dont le caractère névralgique de l'apprentissage du langage oral et écrit au préscolaire et au premier cycle du primaire, du dépistage précoce, du suivi des progrès et du partenariat des services éducatifs et des services complémentaires, et de l'adaptation scolaire pour ce faire. Dans cet esprit, un groupe de développement réunissant des professionnels des services éducatifs et des services complémentaires et de l'adaptation scolaire est mis sur pied. Son mandat est de se donner une vision partagée des apprentissages fondamentaux sur le plan du développement du langage oral et écrit au préscolaire, de pratiques efficaces pour les soutenir et d'outils de suivi des progrès pour les évaluer. Cette même année, le comité du projet éducatif de l'école primaire à l'Orée-du-Bois coordonne l'actualisation de son projet éducatif. À la suite de l'analyse de la situation actuelle des forces et défis de l'école, l'équipe-école et ses partenaires rendent explicite la priorité qu'ils veulent donner à l'accompagnement des élèves à risque et en difficulté. 
En 2012-2013, la direction générale et la directrice des services complémentaires et de l'adaptation scolaire proposent à l'équipe de l'école à l'Orée-du-Bois d'agir en tant qu'école-pilote pour le déploiement d'actions inspirées d'un modèle RàI pour l'apprentissage du langage oral et écrit au préscolaire et au premier cycle du primaire. La communauté d'apprentissage professionnelle (CAP) est retenue comme type d'activité de formation continue par l'équipe du préscolaire et certains professionnels de l'école. Cette même année, le comité de veille CS-RàI est mis sur pied pour réguler le projet-pilote et les différentes ressources humaines, matérielles et financières associées. Ses membres préparent, pour 2013-2014, une première scénarisation annuelle de leurs actions collectives prioritaires. Au printemps 2013, le comité du projet éducatif de l'école à l'Orée-du-Bois fait le bilan de l'an 1 du projetpilote. Les données sur l'état de la réussite des élèves du préscolaire sur le plan du développement du langage oral et écrit et le nombre d'élèves référés en orthopédagogie sont encourageantes. L'équipe-école choisit de poursuivre le déploiement du modèle RàI en $1^{\text {re }}$ année.

En 2013-2014, une CAP en $1^{\text {re }}$ année voit le jour au sein de l'écolepilote. Le groupe de développement poursuit ses travaux pour préciser les apprentissages fondamentaux en $1^{\text {re }}$ année, identifier des pratiques efficaces pour les soutenir et développer des outils de suivi des progrès. Tous accentuent leur développement professionnel et leur réseautage, à l'interne et à l'externe, pour soutenir le comité de veille CS-RàI, le groupe de développement des professionnels et le projet-pilote à l'Oréedu-Bois. L'équipe de conseillers pédagogiques axe son plan de formation continue annuel sur la compétence conjuguée direction d'établissementconseiller pédagogique. Les orthophonistes sont désormais réunies en communauté de pratique pour, notamment, renforcer leur soutien aux actions inspirées d'un modèle RàI dans les écoles. En cours d'année, la direction générale et le comité de veille soutiennent l'actualisation du plan stratégique de la commission scolaire (CSTL, 2014), lequel fait désormais référence à l'essentielle collaboration de tous les partenaires de la réussite pour soutenir les élèves à risque et en difficulté et au modèle RàI comme un moyen privilégié en ce sens.

Plusieurs équipes-écoles témoignent de leur intérêt à mettre sur pied une CAP au préscolaire pour soutenir des actions inspirées d'un modèle RàI. En 2014-2015, 15 écoles adoptent la CAP au préscolaire pour le déploiement d'actions inspirées d'un modèle RàI. L'école-pilote poursuit le déploiement d'actions inspirées d'un modèle de RàI en $2^{\mathrm{e}}$ année. Des données que l'équipe comptabilise sur les effets de leurs 
actions sur le nombre d'élèves référés en orthopédagogie et sur l'état de la réussite des élèves en français sont encourageantes et partagées, à l'interne et à l'externe, aux partenaires de la communauté éducative. Le comité de veille CS-RàI, disparaît. Le déploiement d'actions inspirées d'un modèle RàI devient un objet de chaque comité consultatif de gestion (CCG), qui réunit tous les cadres scolaires de la commission scolaire. Un espace intranet-extranet est crée pour rendre disponibles les informations, les documents et les outils utiles aux professionnels et aux équipes-écoles. Une communauté de pratique des enseignantsorthopédagogues, une communauté de pratique de psychoéducateurs et de techniciens en éducation spécialisée et une autre réunissant les psychologues sont créées. Elles contribuent à une synthèse des rôles des différents partenaires de la réussite dans une école et une commission scolaire en contexte de déploiement d'un modèle RàI. Les directions des services éducatifs et des services complémentaires et de l'adaptation scolaire prennent en charge la communauté de pratique des directions d'établissements nouvellement mise sur pied, la coordination du groupe de développement des professionnels et celle des rencontres de pilotage pour réguler leurs actions d'accompagnement des écoles impliquées dans le projet. Au printemps 2015, en partenariat avec un chercheur universitaire, une demande de financement est effectuée et obtenue dans le cadre du programme de partenariat en adaptation scolaire du ministère de l'Éducation et de l'Enseignement supérieur (MEES). Elle contribue au développement et à la validation des outils de suivi des progrès.

En 2015-2016, 16 écoles travaillent en CAP au préscolaire, en $1^{\text {re }}$ année ou en $2^{\mathrm{e}}$ année. Le groupe de développement des professionnels poursuit ses actions en collaboration avec le chercheur universitaire. Les outils de suivi des progrès sont ainsi validés et progressivement partagés à d'autres commissions scolaires qui en font la demande (Nardonne et Lemieux, 2016; Lupien et Marcoux, 2017).

En 2016-2017, 22 écoles adoptent la CAP comme moyen de formation continue au préscolaire ou en $1^{\text {re }}, 2^{\mathrm{e}}$ ou $3^{\mathrm{e}}$ année pour le déploiement d'actions inspirées d'un modèle RàI. À l'automne 2016, la direction générale et les directions des services acceptent l'invitation de l'Association québécoise des orthopédagogues du Québec pour une diffusion de son modèle d'accompagnement du développement pédagogique et organisationnel pour la mise en œuvre d'actions inspirées d'un modèle RàI (Guay, Francoeur et Ménard, 2016). L'école à l'Orée-du-Bois actualise son projet éducatif en explicitant son souhait 
de transposer ses actions RàI à l'apprentissage de l'écriture et de la compétence sociale des élèves (école à l'Orée-du-Bois, 2017).

En 2017-2018, 22 écoles travaillent en CAP au préscolaire ou en premier, $2^{\mathrm{e}}, 3^{\mathrm{e}}$ ou $4^{\mathrm{e}}$ année pour le déploiement d'actions inspirées d'un modèle RàI. On accentue le soutien au développement du rôle-conseil des professionnels, notamment en contexte de rencontres multidisciplinaires pour soutenir les élèves non répondants à des interventions de palier 2 . Des actions sont également initiées avec des partenaires régionaux pour se donner des outils de collaboration et de communication plus efficaces pour soutenir les élèves qui ont besoin d'interventions de palier 3 . Le rapport annuel 2016-2017 de la Commission scolaire des Trois-Lacs rapporte plusieurs données témoignant de la hausse de la réussite des élèves au préscolaire et au $1^{\text {er }}$ cycle au plan du développement du langage oral et écrit et celle des élèves à risque en français, notamment, lors du passage primaire-secondaire (CSTL, 2017).

\section{Récit de la mise en œuvre de modèles RàI à la Commission scolaire des Hautes-Rivières}

La Commission scolaire des Hautes-Rivières, en Montérégie, dessert plus de 18000 élèves au sein de 35 établissements au primaire, 7 établissements au secondaire, un centre pour la formation générale aux adultes et un autre pour la formation professionnelle où œuvrent plus de 2500 employés. En 2014-2015, la commission scolaire est aux prises avec des enjeux de réussite scolaire et éducative, notamment lors des premières années de scolarisation. De plus, les services en orthopédagogie sont engorgés. La directrice adjointe des services éducatifs et son équipe d'orthopédagogues se posent les questions suivantes : comment agir plus efficacement auprès des élèves à risque et en difficulté d'apprentissage? Comment outiller les enseignants afin qu'ils accompagnent mieux les élèves éprouvant des difficultés dans leurs premiers apprentissages en littératie? Un comité consultatif est mis sur pied. Composé de la directrice adjointe des services éducatifs, de membres du syndicat, de professionnels, de membres du personnel de soutien, de directions d'établissements et de représentants des parents, il a pour mandat de réfléchir sur les moyens qui peuvent être mis en œuvre pour améliorer le modèle de service en adaptation scolaire. C'est dans le cadre de leurs travaux que l'option de s'engager dans un projet systémique du développement d'un modèle $\mathrm{RaI}$ est retenue afin d'agir tôt et autrement pour assurer une meilleure réussite des élèves. 
En 2015-2016, on prépare le terrain. Un comité de pilotage est constitué afin d'approfondir les concepts du modèle RAI et de planifier son déploiement à l'échelle de la commission scolaire. Le comité fait appel à un chercheur universitaire pour le soutenir dans sa réflexion et élaborer un plan d'action réaliste et efficient. La direction générale est partie prenante des réflexions et des décisions du comité de pilotage et en fait une priorité de la planification stratégique 2014-2019 (CSDHR, 2014). Plusieurs actions de sensibilisation sont conduites auprès de toutes les directions d'établissements et des professionnels lors de rencontres collectives afin de soutenir l'adhésion au projet. Un appel est lancé aux équipes-écoles intéressées à participer à la première phase du projet-pilote en tant que communautés d'apprentissage en soutien au déploiement d'un modèle RàI au préscolaire et au premier cycle du primaire. Cette initiative s'inscrit dans le cadre du projet Collaborer, apprendre et réussir (CAR) subventionné par la Fondation Lucie et André Chagnon. Trois écoles acceptent de s'engager dans l'implantation du modèle. Leurs directions se joignent au comité de pilotage afin de préparer la mise en place du projet à la rentrée scolaire 2016. Les équipes-écoles ont accès à un cahier de charges et à un calendrier des opérations pour planifier leurs actions et les différentes ressources humaines, financières et matérielles requises. Un guide et des outils pour démarrer une communauté d'apprentissage (Brochu, Gagnon et Lachapelle, 2016) sont aussi disponibles sur le portail interne de la commission scolaire.

En 2016-2017, on amorce le chantier. Des journées de formation sont proposées aux membres de la cohorte A, c'est-à-dire aux enseignantes, aux directions d'établissements et aux orthopédagogues des trois écoles-pilotes. La formation est offerte par le chercheur qui assume le rôle d'expert de contenus. Elle porte sur les concepts sousjacents à l'apprentissage de la lecture en début de scolarisation afin de soutenir la mise en place du palier 1, c'est-à-dire d'interventions universelles. On y discute notamment des valeurs pédagogiques et des pratiques d'enseignement à ajuster et à introduire pour consolider les apprentissages des élèves. Les orthopédagogues et les orthophonistes de la commission scolaire sont formées sur la nature, les finalités et la mise en œuvre d'un modèle RàI. L'équipe de développement œuvre également à la création d'outils de dépistage avec l'aide du chercheur (Audet, Bazinet et Desrochers, 2016). Les premières collectes de données pour le dépistage des élèves à risque ou en difficulté ont lieu en février 2017. Elles permettent aux équipes des écoles-pilotes de se familiariser avec 
les outils et de les valider avant de les partager à plus grande échelle. En complément aux travaux réalisés par les écoles-pilotes et le comité de pilotage, une communauté d'apprentissage d'orthopédagogues est créée. Elle a pour objectif de développer les outils du palier 3 et de partager le fruit de son travail à l'ensemble des orthopédagogues de la commission scolaire. Au terme de cette première année d'implantation, cinq nouvelles écoles posent leur candidature pour amorcer la mise en place d'un modèle RàI en contexte de communauté d'apprentissage (cohorte B) et trois d'entre elles sont retenues, car elles répondent aux critères de sélection. Une guide d'implantation d'un modèle RàI destiné aux enseignants est désormais disponible pour eux (Bazinet et coll., 2016).

En 2017-2018, on élargit le champ d'action. Le déploiement en est à sa troisième année et amène de grands défis de coordination de ressources, car plusieurs sous-groupes sont en action de façon interdépendante. La cohorte A systématise la mise en place de pratiques universelles et le dépistage, et elle amorce l'élaboration de son modèle d'intervention au palier 2. La cohorte B expérimente les outils de dépistage élaborés par la cohorte A afin de les bonifier. Ces outils permettent de mieux suivre l'évolution des apprentissages des élèves du préscolaire et du premier cycle dans chaque école et, éventuellement, à l'échelle de la commission scolaire. Également, la cohorte B approfondit sa réflexion sur la progression des objets d'apprentissage priorisés.

On prend la décision de dissoudre le comité consultatif et le comité de pilotage. Le premier est remplacé par un comité de vigie où siège désormais la direction générale. Il a le mandat de garder le cap sur la vision et sur les différentes situations pédagogiques interreliées. Il doit aussi prévoir le déploiement, à long terme, du modèle dans l'ensemble des écoles primaires volontaires. Le comité de pilotage est remplacé par le comité de coordination, qui développe notamment un schéma rendant visible l'interdépendance, les rôles et la contribution des différents comités et communautés en soutien au déploiement du modèle RAI (Poirier, 2017). L'équipe de développement se réunit chaque semaine. Elle assure un suivi attentif des actions de développement, de recherche et de formation nécessaires au déploiement du modèle RàI dans les écoles. La communauté des orthopédagogues, composée de six dyades provenant des différents secteurs de la commission scolaire, poursuit le développement d'outils d'évaluation communs pour intervenir au palier 3. Ces dyades ont également pour mandat de soutenir les orthopédagogues de leur secteur dans l'appropriation 
et l'expérimentation de ces outils. Des rencontres collectives avec les directions d'école engagées dans les cohortes A et B sont prévues à l'agenda annuel afin de leur permettre d'aborder de façon plus spécifique des questions relatives à la gestion concernant, notamment, l'accompagnement individuel et collectif des membres de leur équipe. Un guide de pilotage du modèle RAI est élaboré pour les soutenir dans leur rôle et fonction (Poirier, 2018). Au terme de ces deux années d'apprentissages, d'actions et de recherche, le bilan des impacts du projet sur le développement professionnel des enseignants est encourageant. Ils affirment mieux comprendre les difficultés des élèves et leur source, être plus conscients des stades de développement de la lecture chez les élèves et avoir haussé leur compétence à mener une activité de dépistage et à organiser des interventions supplémentaires et ciblées.

\section{De bons coups dans la mise en œuvre de modèles RàI}

Dans nos milieux respectifs, la mise en œuvre de modèles RàI inspirée d'un modèle d'accompagnement systémique du développement pédagogique et organisationnel a été marquée par certains bons coups, dont les suivants :

\section{La précision des concepts et des présupposés sous-jacents à la mise en ouvre d'un modèle RàI}

Qu'est-ce qu'un modèle RàI? Quelles sont les pratiques universelles à instaurer en classe? Faut-il ou non enseigner les lettres de l'alphabet au préscolaire? Qu'est-ce qu'un outil valide de suivi des progrès? Quel type d'activité de formation continue privilégier pour soutenir le développement professionnel des directions d'établissements sur l'animation d'une communauté? Des réponses claires et consensuelles à de telles questions concernant les composantes et les relations des situations pédagogiques constitutives d'une organisation scolaire sont névralgiques en contexte de déploiement d'un modèle RàI. Elles exigent que les équipes de leaders partagent et négocient leurs conceptions et leurs présupposés au regard de ce qu'est un modèle RàI et de tous les autres concepts qui lui sont associés sur le plan de l'apprentissage, de l'enseignement, de l'évaluation, de la collaboration, etc. Nos équipes ont ainsi produit des documents de référence pour soutenir, d'une même voix, le développement des élèves, des équipes-écoles, des directions d'établissements et des professionnels en rôle-conseil dont, par exemple, 
une liste de pratiques efficaces pour soutenir le développement du langage oral et écrit au préscolaire/premier cycle du primaire et un guide précisant la nature, les finalités et le processus de mise en œuvre d'une CAP.

\section{Le projet-pilote pour agir, éduquer et chercher}

En quoi la mise en œuvre d'un modèle RàI est-elle congruente avec nos projets antérieurs? En quoi constitue-t-elle un levier complémentaire pour soutenir l'état de la réussite de nos élèves? Qui serait favorable à s'engager dans un projet-pilote sachant qu'il implique une hausse de la collaboration et de l'innovation? Quel est notre cadre de référence pour baliser un tel projet-pilote et en évaluer les impacts? Dans nos deux milieux, des projets-pilotes du déploiement d'un modèle RàI ont été initiés, à petite échelle, avant d'être étendus à d'autres milieux. Ils ont d'abord mobilisé une ou quelques écoles et certains gestionnaires et professionnels proactifs dont les actions, le développement professionnel et les recherches ont été évalués attentivement. Pour ce faire, les grandes étapes de la recherche-action ont servi de cadre de référence, c'est-à-dire que les équipes de leaders ont eu le souci :

1) d'analyser leur situation actuelle;

2) de cibler un ou quelques objectifs/indicateurs prioritaires découlant de cette analyse;

3) d'établir un plan de leurs actions assorti d'un échéancier;

4) de le mettre en œuvre avec souplesse;

5) d'évaluer l'impact de leurs actions; et

6) de diffuser les connaissances praxéologiques et les apprentissages issus de leurs actions.

Dans les deux milieux, plusieurs connaissances praxéologiques ont ainsi été développées et ont servi au déploiement progressif d'actions inspirées d'un modèle RàI, dont une synthèse des apprentissages fondamentaux au plan du développement du langage oral et écrit au préscolaire et au premier cycle du primaire, des trousses d'outils de suivi des progrès pour le préscolaire, la $1^{\text {re }}$ année et la $2^{\mathrm{e}}$ année du $1^{\mathrm{er}}$ cycle du primaire, un modèle de scénarisation annuelle des moyens prioritaires RàI des équipes de professionnels et des modèles de déploiement d'un modèle RàI au sein d'une commission scolaire. 


\section{Les essentielles activités de formation continue de nature collaborative}

Que voulons-nous que les élèves apprennent? Comment saurons-nous s'ils l'ont appris? Que ferons-nous s'ils ne l'apprennent pas? Qu'est-ce que les enseignantes-orthopédagogues ont besoin d'apprendre pour soutenir le déploiement d'un modèle RàI au préscolaire? Quelles sont nos ressources humaines, financières et matérielles actuelles pour soutenir le déploiement d'actions inspirées d'un modèle RàI? Dans nos milieux, ces questions fondamentales sont traitées, dans l'action, dans le cadre d'activités de formation continue de nature collaborative, c'est-à-dire, entre autres, en contexte de communauté d'apprentissage professionnelle, de communauté d'apprentissage, de communauté de pratique, de groupe de développement, d'accompagnement soutenu et de rencontre multidisciplinaire auxquelles nous avons associé une définition spécifique.

Toutes ces activités de formation continue, de nature collaborative, ont en commun d'offrir aux équipes de leaders un espace récurrent de dialogue et de partage, dans le quotidien, pour agir, s'éduquer et chercher sur l'apprentissage du langage oral et écrit. Autant les équipes-écoles, les directions d'établissements et les professionnels que les cadres des services et la direction générale participent à de telles activités de formation continue qui, aussi souvent que possible, rassemblent des leaders aux présupposés et aux rôles complémentaires. En plus de contribuer au développement individuel et collectif au sein de l'organisation, ces activités soutiennent la création de sens communs, la mobilisation et la résolution de problèmes.

\section{Une vision organisationnelle explicite et cohérente avec le modèle RàI}

En quoi le déploiement d'un modèle RàI constitue-t-il une priorité organisationnelle, à moyen et à long terme, laquelle influencera nos priorités d'actions et l'attribution de nos ressources? La vision d'une organisation scolaire est idéalement le fruit d'un travail de codéfinition d'une équipe de leaders. Elle est notamment perceptible à travers ses plans généraux (plan stratégique, plan d'engagement vers la réussite, projet éducatif), ses plans d'action annuels, ses politiques, son rapport annuel et ses présentations collectives aux équipes. Dans nos deux milieux respectifs, au sein de la commission scolaire comme au sein des écoles, des présupposés, des orientations et des objectifs, 
en cohérence avec le modèle RàI, ont été progressivement précisés et rendus explicites dans de tels documents. Par exemple, des mentions relatives à la différenciation pédagogique, à la collaboration et au soutien à la réussite de tous, dont les élèves à risque et en difficulté, y sont apparues. La mise en œuvre d'un modèle RàI et des ajustements sur le plan de l'enseignement, de l'évaluation et de la gestion y ont aussi été mentionnés comme moyens pour atteindre de tels objectifs. On a agi ainsi pour faire en sorte que ce développement pédagogique et organisationnel soit ancré dans la vision organisationnelle et qu'il contribue à l'enrichir, par-delà les modes pédagogiques, les mouvements du personnel et les changements d'alignements ministériels.

\section{La technologie pour archiver, partager et communiquer efficacement}

Où se trouve la synthèse des pratiques efficaces pour l'enseignement du vocabulaire des élèves résistant aux interventions de palier 2? Comment s'assurer que toutes les écoles aient accès à la version actualisée de l'outil de suivi des progrès du printemps pour le préscolaire? Où se trouve le compte rendu de la dernière rencontre de la CAP et les traces de nos décisions pour soutenir ces élèves? Quelle est la date de la prochaine rencontre du comité de vigie où nous pourrions clarifier cette question? Le déploiement d'un modèle RàI a rapidement nécessité la mise en œuvre d'un espace virtuel pour partager des textes, des référentiels et des outils créés de même que des traces des échanges et des choix effectués. Office 365 , qui permet le travail collaboratif sur des documents, leur archivage et leur accès à des personnes spécifiques, à l'intérieur et l'extérieur de l'organisation, s'est avéré d'une grande utilité. Les équipes-écoles, les différents comités et les communautés contribuant au déploiement de modèles RàI se sont ainsi donné des espaces intranet ou extranet leur permettant, en temps réel, de mettre à jour, partager, organiser tout ce dont ils ont besoin pour agir, apprendre, communiquer et chercher efficacement.

\section{Des outils et des espaces pour voir et faire voir nos actions et leurs impacts}

Quelles sont les actions annuelles prioritaires de l'équipe du préscolaire pour soutenir la mise en œuvre d'un modèle RàI? Celles des directions des services et du groupe de développement? À ce jour, quels sont les impacts de nos efforts collectifs sur la réussite en français de nos élèves à risque? 
En quoi l'équipe des psychologues scolaires contribue-t-elle cette année au déploiement du modèle à l'échelle de l'organisation? Dans nos deux milieux respectifs, plusieurs référentiels sont créés pour permettre aux équipes de leaders de rendre explicite régulièrement ce qu'ils vivent et permettre aux autres de le voir aussi, dont une scénarisation annuelle des actions prioritaires RàI des professionnels des services éducatifs et de l'adaptation scolaire, une représentation graphique des liens unissant les différentes communautés en soutien aux développements RàI, une synthèse des rôles des différents membres d'une équipe-école impliqués dans la mise en œuvre d'un modèle RàI ainsi que le rapport annuel de l'école et de la commission scolaire. Ils permettent à tous les membres de l'organisation de voir, de façon synthétique, les actions prioritaires en cours en lien avec le déploiement d'actions RàI et leurs impacts. Les équipes de leaders ont également le souci de multiplier les espaces où on réfléchit et on discute d'actions liées à la mise en œuvre RàI. Par exemple, chaque année, en lien avec son projet éducatif, l'équipe-école de l'école-pilote de la CSTL établit et régule ensemble la liste d'une dizaine de moyens prioritaires à mettre en œuvre pour soutenir la réussite, dont ceux associés à la mise en œuvre d'un modèle RàI. Cette question est également un objet de planification, de régulation et de partage des rencontres des directions de services, des directions d'établissements, des équipes de professionnels, etc. En se donnant de tels outils et espaces pour voir et faire voir les actions RàI et leurs impacts, les équipes de leaders témoignent de l'importance de ces actions, leur donnent progressivement un sens commun et peuvent en optimiser ensemble l'efficacité.

\section{Des leaders authentiques qui sont accompagnés pour agir, apprendre, éduquer et chercher}

En tant que direction générale, direction de service, direction d'établissement, professionnel, personnel de soutien, enseignant ou orthopédagogue, en quoi incarnons-nous la prise en compte de la différence, la collaboration et l'innovation? En quoi sommes-nous engagés dans notre développement professionnel pour inspirer les autres à apprendre sur un modèle RàI? Par qui sommes-nous accompagnés en ce sens? Dans nos milieux, plusieurs équipes de leaders témoignent d'un souci de leadership authentique, lequel est névralgique pour le déploiement d'actions inspirées d'un modèle RàI. Elles cherchent elles-mêmes à agir et à éduquer en cohérence avec les présupposés et les actions sous-jacentes à un modèle RàI, c'est-à-dire à accueillir la différence, 
à collaborer pour la prendre en compte, à prioriser leur propre développement professionnel et celui de leurs équipes et à oser faire autrement au service de la réussite. Des actions, dont les suivantes, en témoignent : la création d'un comité de veille du plan stratégique ou du projet éducatif pour définir, réguler et évaluer les actions des équipes, la création d'une fonction inédite pour l'accompagnement des directions et des équipes-écoles et le transfert des connaissances, la mise en place d'une équipe de recherche et développement, la participation active à des activités de formation et de diffusion des connaissances praxéologiques générées et la conception d'un modèle d'accompagnement systémique du développement pédagogique et organisationnel.

\section{Des défis dans la mise en œuvre de modèles RàI}

Dans nos milieux, la mise en œuvre de modèles RàI, inspirée d'un modèle d'accompagnement systémique du développement pédagogique et organisationnel, a également été caractérisée par certaines difficultés explicitées ci-dessous.

\section{L'inévitable ajustement des rôles de chacun}

Est-ce à l'orthopédagogue ou à l'enseignante d'enseigner la conscience phonologique au préscolaire? Le développement d'un modèle RàI est-il une responsabilité des services éducatifs ou des services de l'adaptation scolaire? Que fait concrètement un psychologue scolaire en soutien à la mise en œuvre d'une CAP dans une école? Quels sont les rôles et les responsabilités d'une direction d'établissement et d'un conseiller pédagogique dans le cadre d'une CAP? En quoi est-il du ressort d'une direction des services éducatifs d'accompagner le développement professionnel des directions d'établissements? La mise en œuvre d'un modèle RàI vient modifier, de façon tangible, les rôles de chacun dans le soutien à la réussite des élèves. Tous doivent accentuer leur collaboration pour se donner une vision partagée des objets de développement et des pratiques universelles et différenciées à mettre en œuvre. Ils doivent aussi collaborer pour développer des outils essentiels à leurs actions complémentaires de soutien aux élèves à risque et en difficulté, lequel passe par la consolidation de la réussite pour tous. Nos milieux respectifs ont vu naître de larges inconforts chez le personnel scolaire impliqué dans la mise en œuvre d'un modèle RàI. Ceux-ci ont été abordés le plus ouvertement possible dans le cadre des différentes activités de 
formation continue de nature collaborative. Certains documents ont été produits pour décrire divers rôles qui se modifiaient et continueront à se modifier au fil de l'évolution de la mise en œuvre du modèle RàI. Certaines personnes ont changé de poste ou d'école ou ont dû être accompagnées de près pour soutenir cette transition identitaire au plan professionnel.

\section{L'accès aux connaissances issues de la recherche}

Comment mobiliser l'équipe du préscolaire au regard de l'enseignement des lettres de l'alphabet alors que le programme de formation en vigueur est circonspect sur cette question? À qui faire appel pour accéder à une synthèse exhaustive des pratiques démontrées les plus efficaces pour soutenir le développement de la conscience phonologique? Comment collaborer efficacement avec un chercheur universitaire? En quoi un modèle RàI de type "résolution de problème " est-il différent et plus ou moins efficient qu'un modèle RàI de type "protocole standard "? La mise en œuvre d'un modèle RàI exige qu'une équipe de leaders se donne une vision précise des objets d'apprentissages des élèves, des enseignants, des orthopédagogues, des professionnels et des directions d'établissements ainsi que des pratiques efficaces pour les soutenir. Or, à ce jour, les équipes de leaders ont peu de temps, de préalables et d'outils pour accéder à de telles synthèses. Qui plus est, elles doivent composer avec de nombreux débats et interrogations suscités par des écarts substantiels entre l'état de la recherche et les programmes d'études en vigueur ainsi que des tensions ouvertes entre certains groupes de chercheurs aux présupposés différents sur le plan de l'apprentissage du développement du langage oral et écrit. Lorsque certains chercheurs acceptent de collaborer avec les équipes de leaders, la nature de leurs engagements universitaires, leur horaire et la distance amènent des défis substantiels au plan opérationnel. Il s'agit là d'entraves importantes à l'accès aux connaissances essentielles au développement pédagogique et organisationnel inhérent à la mise en œuvre d'un modèle RàI.

\section{La création d'espaces de collaboration de qualité}

Quand vivre la CAP pour éviter de multiplier les libérations et les frais associés? Comment organiser la participation de l'orthopédagogue à la CAP sans trop réduire son temps d'implication dans les interventions au palier 3? Comment assurer la participation efficace de la direction d'établissement à la CAP dans une école qui vit plusieurs CAP à 
plusieurs niveaux? Comment s'organiser pour que les directions des services éducatifs et de l'adaptation scolaire aient le temps de prendre $d u$ recul et être proactives sur les développements RàI en cours? La nécessaire collaboration inhérente à la mise en œuvre d'un modèle RàI appelle la création d'espaces de qualité pour ce faire. Cela pose un défi de taille dans nos milieux respectifs et nécessite beaucoup d'écoute, de patience et de créativité. Certaines sommes ont été décentralisées par les services éducatifs pour soutenir les frais de libération des enseignants, mais la rareté de suppléants vient contrecarrer cette mesure. Une synthèse des modèles de communautés a été produite et partagée entre les acteurs scolaires. Elle a plusieurs fois fait l'objet de recherche et d'échanges dans le cadre de la communauté de pratique des directions d'établissements. Des équipes se sont donné des modalités et des critères pour approfondir certains échanges au regard de certains élèves particuliers avant ou après les rencontres de la communauté. En ce sens, plusieurs directions d'établissements accentuent leurs rencontres individuelles avec les membres de leur équipe-école. D'autres ont créé des modèles d'ordres du jour inédits pour rendre plus opérationnels leurs échanges et la consignation de leurs décisions dans leurs communautés. Dans nos milieux, cet enjeu est notamment à l'origine d'un processus de recherche de fonds complémentaires par le biais de demandes de subventions auprès du ministère de l'Éducation et de la Fondation Chagnon dans le cadre du projet CAR. Les sommes ont largement été relayées aux écoles pour soutenir l'aménagement d'espaces collaboratifs inédits tenant compte de leurs caractéristiques et de leurs ressources spécifiques. À l'échelle de la commission scolaire, les directions des services éducatifs se sont donné des espaces de réflexion où elles sont accompagnées de la coordonnatrice au développement ou de la conseillère en gestion de l'éducation. Ce sont notamment des moments privilégiés pour discuter de la cohérence des présupposés sous-jacents au développement pédagogique et organisationnel en cours. Inspirées de l'adage «On va plus vite tout seul, on va plus loin ensemble ", elles veulent ainsi se donner les conditions pour faire bien, efficacement, dans le respect de soi et des autres.

\section{Conclusion}

À la Commission scolaire des Trois-Lacs et à la Commission scolaire des Hautes-Rivières, un modèle d'accompagnement systémique du développement pédagogique et organisationnel guide le déploiement de modèles RàI inédits relatés ici sous la forme de récits. Ces récits mettent 
en lumière les actions fondamentales que deux équipes de leaders ont mises en œuvre pour soutenir simultanément le développement des élèves, des équipes-écoles, des directions d'établissements et des professionnels exerçant un rôle-conseil, incluant le leur, en contexte de déploiement d'un modèle RàI. Pour ce faire, on observe que ces équipes veillent à se donner et à rendre explicite une vision systémique des situations pédagogiques qu'elle accompagne et des présupposés qui les sous-tendent. Or, leurs actions sont souvent complexifiées par l'accessibilité des synthèses des connaissances issues de la recherche et les débats idéologiques qui leur sont associés. Également, on observe que ces équipes font le choix de favoriser des projets-pilotes et de créer un réseau d'espaces collaboratifs et des outils de communication novateurs pour vivre et faire voir leurs actions d'éducation et de recherche. De telles façons de contribuer au développement des élèves et de l'organisation viennent toutefois modifier les rôles traditionnels des partenaires de la réussite. Elle exige des équipes de leaders beaucoup de patience, de créativité et de réseautage pour incarner auprès de leurs équipes la différenciation qu'elles veulent voir naître dans les classes pour contribuer à la réussite de chaque élève. On remarque enfin que ces équipes de leaders se sont donné un modèle d'accompagnement systémique pour agir et prendre du recul sur leurs actions. Or, une telle conception du développement pédagogique et organisationnel, soustendue de présupposés favorables au regard de la collaboration, de l'innovation et de la différenciation, est, à ce jour, parfois incompatible avec celle de certaines organisations ou celle de leurs dirigeants. Les équipes de leaders gagnent alors à être accompagnées et supervisées par des partenaires et des gestionnaires sensibles aux présupposés semblables aux leurs pour oser être et faire différemment en soutien au développement individuel et collectif. Nous sommes conscientes que de telles observations sont largement tributaires des caractéristiques des situations pédagogiques interreliées de nos organisations. Nous souhaitons néanmoins qu'elles puissent inspirer les réflexions et les actions d'équipes de leaders qui œuvrent dans des organisations aux présupposés et aux caractéristiques semblables pour amorcer ou poursuivre leur propre mise en œuvre de modèles RàI en soutien à la réussite et au bien-être de tous.

\section{Références}

Audet, R., Bazinet, J. et Desrochers, A. (2016). Trousses de dépistage continu : préscolaire, $1^{r e}$ année, $2^{e}$ année. Services des ressources éducatives aux 
jeunes. Commission scolaire des Hautes-Rivières, St-Jean sur Richelieu, Québec, Canada.

Bazinet, J.(2016). Guide d'implantation du modèle de réponse à l'intervention (RàI) à l'intention des écoles-pilotes. Services des ressources éducatives aux jeunes. Commission scolaire des Hautes-Rivières, St-Jean-surRichelieu, Québec, Canada.

Brochu, D., Gagnon, B. et Lachapelle, D. (2016). Guide de démarrage des différents types de communautés à la CSDHR. Services des ressources éducatives aux jeunes. Commission scolaire des Hautes-Rivières, St-Jean sur Richelieu, Québec, Canada.

Commission scolaire des Hautes-Rivières. (2014).Plan stratégique 2014-2019. http://www.csdhr.qc.ca/?64F3AC2E-8975-4C66-BDBC-54D08884FA8E

Commission scolaire des Trois-Lacs. (2005). Politique sur la formation continue du personnel enseignant. http://www.cstrois-lacs.qc.ca/commissionscolaire/reglements-et-politiques

Commission scolaire des Trois-Lacs. (2014).Plan stratégique 2014-2019. http:// www.cstrois-lacs.qc.ca/commission-scolaire/reglements-et-politiques

Commission scolaire des Trois-Lacs. (2017). Rapport annuel 2016-2017. http://www.cstroislacs.qc.ca/images/documents/Rapports_annuels/ Rapport_annuel_2016-2017_Trois-Lacs.pdf

Commission scolaire des Trois-Lacs, Services complémentaires et adaptation scolaire. (2013). Politique relative à l'organisation des services éducatifs aux élèves à risque et aux élèves handicapés ou en difficulté d'adaptation ou d'apprentissage. http://www.cstrois-lacs.qc.ca/commission-scolaire/ reglements-et-politiques

CTREQ, RENARD et Boily, É. (2015, mars). Identification et documentation de rôles associés à celui d'agent d'intermédiation de connaissances et de réseaux dans le domaine de l'éducation. http://www.equiperenard.ca/lerole-de-lagent-intermediaire-en-milieu-scolaire/

École à l'Orée-du-Bois. Projet éducatif 2017-2022. http://www.cstrois-lacs. qc.ca/ecoles/primaire/oree-du-bois/projet-educatif-et-plan-de-reussite

Guay, M.-H., Francoeur, N. et Daoust, F. (2014, février). Illustration du rôle d'un intermédiaire en soutien au développement pédagogique et organisationnel au sein d'une commission scolaire. Présentation au $2^{\mathrm{e}}$ symposium sur le transfert des connaissances en éducation organisé par le Centre de transfert pour la réussite éducative du Québec (CTREQ), Sainte-Foy, Québec, Canada.

Guay, M.-H., Ste-Marie, I., McSween, A., Bédard, S. et Lecompte, P.-Y. (2015, avril). Parce que nous avons aussi besoin d'apprendre et d'être soutenus au cour de l'action. Présentation au congrès de la Fédération québécoise des directions d'établissement d'enseignement (FQDE), Québec, Canada.

Guay, M.-H., Smith, S., Ménard, L. et Dewany, S. (2015, mai). Nos leviers pour soutenir la réussite : Quels sont-ils? Comment sont-ils visibles 
dans notre quotidien, d'hier à aujourd'hui? Présentation au colloque de l'Association des directeurs généraux des commissions scolaires (ADIGECS), Sainte-Foy, Québec, Canada. http://adigecs.qc.ca/congres/ congres-anterieurs/les-ateliers/

Guay, M.-H., Francoeur, N. et Ménard, L. (2016, octobre). L'accompagnement des écoles dans la mise en œuvre du modèle de réponse à l'intervention. Présentation au colloque de l'Association des orthopédagogues du Québec (ADOQ), Lévis, Québec, Canada.

Guay, M.-H. et Gagnon, B. (2020a). Un modèle d'accompagnement systémique du développement pédagogique et organisationnel pour soutenir la mise en œuvre d'un modèle de réponse à l'intervention, Enfance en difficulté, ce numéro.

Herr, K. et Anderson, G. L. (2015). The Action Research Dissertation : A Guide for Students and Faculty. Thousand Oaks : Sage.

Lachapelle, D., Ménard, N. et Gagnon, B. (2016, mai). La communauté d'apprentissage, un dispositif de développement professionnel systémique au sein d'une commission scolaire. Communication présentée au congrès annuel de l'ACFAS, Montréal, Québec, Canada.

Lupien, J. et Marcoux, S. (2017, octobre). Comment traiter les données de dépistage dans le cadre d'une communauté d'apprentissage professionnelle. Présentation au colloque de l'Association des orthopédagogues du Québec (ADOQ), Laval, Québec, Canada.

Nardone, S. et Lemieux, K. (2016, octobre). Le pilotage de l'implantation du modèle de la réponse à l'intervention: Obstacles et facilitateurs dans la mise en œuvre d'un système éducatif. Présentation au colloque de l'Association des orthopédagogues du Québec (ADOQ), Lévis, Québec, Canada.

McNiff, J. (2016). You and Your Action Research Project. New York : Routledge. https://doi.org/10.4324/9781315693620

Poirier, F. (2017). Structure du projet-pilote RàI 2017-2018. Services des ressources éducatives aux jeunes. Commission scolaire des HautesRivières, St-Jean sur Richelieu, Québec, Canada.

Poirier, F. (2018). Guide de pilotage d'une école RAI. Services des ressources éducatives aux jeunes, Commission scolaire des Hautes-Rivières, St-Jean sur Richelieu, Québec, Canada. 


\section{Correspondance :}

Marie-Hélène Guay

Département de la gestion de l'éducation et de la formation

Université de Sherbrooke (Campus de Longueuil)

150, Place Charles-Le Moyne

Longueuil (Québec) J4K 0A8

Courriel : marie-helene.guay@usherbrooke.ca

\section{Note des auteures}

Marie-Hélène Guay a été coordonnatrice au développement et à la planification stratégique de 2009 à 2017 à la Commission scolaire des Trois-Lacs et Brigitte Gagnon est conseillère pédagogique en gestion de l'éducation à la Commission scolaire des Hautes-Rivières. Elles remercient chaleureusement Lyne Ménard, directrice générale adjointe, Nadine Francœur, directrice des services éducatifs, et Julie Lupien, directrice adjointe des services éducatifs de la Commission scolaire des Trois-Lacs, ainsi que Dominique Lachapelle, directrice générale, Nathalie Rousseau et Fanie Poirier, ex-directrices adjointes aux services éducatifs, et Ruth Phaneuf, ex-directrice d'une écolepilote de la Commission scolaire des Hautes-Rivières, pour leur collaboration à la préparation du présent article.

\section{Résumé}

$\mathrm{Au}$ cours des dernières années, deux commissions scolaires de la Montérégie, au Québec, ont amorcé la mise en œuvre d'un modèle de réponse à l'intervention (RàI) pour l'apprentissage du langage oral et écrit, au préscolaire et au premier cycle du primaire, en s'inspirant d'un modèle d'accompagnement systémique du développement pédagogique et organisationnel (Guay et Gagnon, 2020a). Dans cet article, nous présentons les récits de ces deux expériences de mises en œuvre en détaillant des outils et des dispositifs élaborés pour les appuyer. Nous faisons également état de bons coups et d'obstacles rencontrés. Ces récits visent à guider les équipes de leaders chargées de soutenir le déploiement d'un modèle de réponse à l'intervention dans une organisation scolaire. 
Mots-clés : modèle de réponse à l'intervention; modèle d'accompagnement systémique; développement professionnel et organisationnel; langage oral et écrit; préscolaire; primaire.

\section{Abstract}

In recent years, two school districts in the Montérégie region in Quebec have implemented a response-to-intervention model to support the development of literacy in kindergarten and the first two years of the elementary grades. This implementation was guided by the coaching model developed by Guay and Gagnon (this issue). A narrative account of the application of this coaching model in these two school districts and the development of tools and teaching material are presented in the present article. Finally, we summarize the gains and obstacles encountered in this project. This account is intended to offer some guidance to educational leaders in charge of implementing a responseto-intervention model.

Keywords: response-to-intervention model; coaching model; professional and organizational development; oral and written language instruction in kindergarten and early elementary grades. 